\title{
Hipotermia en el trauma craneoencefálico: algunos aspectos básicos
}

\section{Hypothermia in the traumatic brain injury: some basic aspects}

\author{
Juan Luis Pinedo Portilla ${ }^{1 a}$
}

Sr. Editor: El trauma Craneoencefálico (TEC) es una de las principales causas de muerte y discapacidad a nivel mundial, en especial en la población menor de 45 años. Muchos de los que sobreviven al trauma quedan con secuelas invalidantes que impiden su retorno a la vida productiva, a un costo enorme tanto personal y familiar como para la sociedad. Muchos han sido los tratamientos propuestos para el manejo del TEC con el afán de mejorar la tasa de sobrevida y el resultado neurológico final. Dentro de estos, la hipotermia es una de las terapias que ha generado gran interés y al mismo tiempo gran controversia debido a los resultados obtenidos de los grandes estudios multicéntricos ${ }^{(1)}$.

Para entender el tema debemos conocer algunos aspectos básicos acerca del mismo. Como sabemos la temperatura corporal normal es de $36,8^{\circ} \mathrm{C}$ en promedio (medida en la cavidad oral) con una variación diurna de $0,5^{\circ} \mathrm{C}$. Esta temperatura es regulada por el equilibrio entre la producción (metabolismo basal, actividad muscular, actividad hormonal, etc.) y la perdida de calor ${ }^{(1)}$.

La hipotermia terapéutica se define como una temperatura corporal central inducida y controlada inferior a $36,0^{\circ} \mathrm{C}$, con logros terapéuticos predefinidos y tiempos predeterminados ${ }^{(2)}$; pero antes aclaremos dos términos en esta presente carta al editor:

1) Hipotermia profiláctica (HP), la cual hace referencia a la hipotermia que se aplica inmediatamente después del TEC con el objetivo de neuroprotección al disminuir la lesión secundaria mediante la atenuación de los procesos inflamatorios temperatura-sensibles.

2) Hipotermia terapéutica (HT), la cual es una medida de manejo de segunda línea en la hipertensión endocraneana luego que las medidas de primera línea han fallado.

Este último término de hipotermia terapéutica fue cambiado en el 2009 al de "Manejo de la temperatura objetivo" (en inglés: targeted temperature management); dicho cambio se realizó en una conferencia internacional realizada en la ciudad de San Juan de Puerto Rico donde participaron cinco sociedades de cuidado intensivo, a saber, Society of Critical Care Medicine (SCCM), European Society of Intensive Care Medicine (ESICM), American Thoracic Society (ATS), European Respiratory Society (ERS) y Société de Réanimation de Langue Francaise (SRLF), en la cual se trataron varios aspectos relacionados a la hipotermia en el ambiente del cuidado intensivo.

El motivo por el cual fue considerado inadecuado fue el hecho de que el adjetivo "terapéutico" implicaba un efecto favorable lo cual no está demostrado aún y por el ultimo el término "hipotermia" no engloba algunas intervenciones cuyo objetivo es mantener la temperatura corporal cercana a $37^{\circ} \mathrm{C}$, la cual es considerada una temperatura "normal" (3).

Dentro de los efectos terapéuticos a nivel cerebral se incluyen: diminución del metabolismo cerebral, pr ducción de radicales, acidosis intracelular, neurotransmisores excitatorios, reacciones enzimáticas destructivas

\footnotetext{
1. Hospital Nacional Almanzor Aguinaga Asenjo, Lambayeque, Perú.

a. Médico especialista en Medicina Intensiva
} 
e inhibición de la apoptosis ${ }^{(3)}$. Este procedimiento consta de tres fases: inducción, mantenimiento y recalentamiento. Estas fases aún están en debate pues no hay una recomendación clara sobre qué tan rápido se debe enfriar o cuanto tiempo es el que debe estar sometido a hipotermia o que tan rápido debe ser recalentado.

A pesar de la gran cantidad de estudios preclínicos que han indicado los efectos beneficiosos de la HT, no se han descrito intervenciones clínicas exitosas que demuestren un claro efecto benéfico de la HT sobre la población con TEC, esto es mencionado en una revisión realizada por Cochrane en 2017 por Sharon R. Lewis y Colaboradores ${ }^{(4)}$. Asimismo, otras revisiones han buscado evidencia sobre los efectos de la hipotermia en pacientes operados de aneurismas cerebrales, no pudiendo, igualmente, observar un claro efecto benéfico de la misma ${ }^{(5)}$.

Estos hallazgos podrían explicarse por la inclusión de diversos tipos de lesiones en protocolos de tratamiento ampliamente divergentes. Es deseable seleccionar una entidad patológica más homogénea para un tratamiento uniforme; no obstante, aun así el manejo de la temperatura, como el evitar la fiebre en el paciente neurocrítico, sigue siendo un pilar en la práctica clínica para TEC severo ${ }^{(4-7)}$.

\section{REFERENCIAS BIBLIOGRÁFICAS}

1. Kochanek $P$, Jackson T. The Brain and Hypothermia-From Aristotle to Targeted Temperature Management. Crit Care Med. 2017; 45(2):305-10.

2. Ugarte S. Hipotermia y Termomodulación en urgencias y paciente crítico. Editorial Distribuna. 2017. Colombia. 28p.

3. Nunnally ME, Jaeschke R, Bellingan GJ, Lacroix J, Mourvillier B, Rodriguez-Vega GM, et al. Targeted temperature management in critical care: a report and recommendations from five professional societies. Crit Care Med. 2011; 39(5):1113-25.

4. Lewis SR, Evans DJW, Butler AR, Schofield-Robinson OJ, Alderson P Hypothermia for traumatic brain injury. Cochrane Database of Systematic Reviews 2017, Issue 9. Art. No.: CD001048. DOI: 10.1002/14651858. CD001048.pub5

5. Neeraj B. Hyperthermia and fever control in brain injury. Crit Care Med. 2009; 37(7suppl):S250-7.

6. Lasry O., Schur S., Hawryluk, G: Prophylactic and Therapeutic Hypothermia in Severe Traumatic Brain Injury. Curr Trauma Rep. 2018; 4(2):109-120.

7. Li L, You C, Chaudhary B. Intraoperative mild hypothermia for postoperative neurological deficits in people with intracranial aneurysm. Cochrane Database of Systematic Reviews 2016, Issue 3. Art. No.: CD008445. DOI: 10.1002/14651858.CD008445.pub3.

Revisión de pares: Recibido: 05 / 02 / 2019 Aceptado: 21/ 03/ 2019 